\title{
NERI MOXERIFFO, CONSOLE DEI CATALANI A CASTEL DI CASTRO NELL'ANNO $1320^{1}$
}

\author{
MARIA EUGENIA CADEDDU \\ Istituto sui Rapporti Italo-Iberici \\ (CNR, Cagliari, Italia)
}

In una lettera datata 9 marzo 1301 e indirizzata a Giacomo II d'Aragona i consiglieri della città di Barcellona si rammaricavano con il loro sovrano perché questi, venendo meno a quanto stabilito da un privilegio del suo predecessore Giacomo I, privilegio da lui stesso confermato, aveva autonomamente eletto un pisano console dei Catalani a Castel di Castro, mentre essi avevano già provveduto a designare per tale carica il barcellonese Ramon de Tolosa².

I consiglieri naturalmente non si limitavano a segnalare l'equivoco al re in modo diplomatico, "credimus quod hoc fecistis de nostro privilegio et confirmacione tunc memoriam non habente", ma con devozione, e altrettanta determinazione, gli chiedevano di revocare il provvedimento e di rispettare in futuro quanto disposto in loro favore da Giacomo I riguardo all'elezione dei consoli d'Oltremare.

\footnotetext{
'Desidero esprimere la mia più sincera gratitudine alla dott. Josefina Mutgé Vives e al dott. James W. Nelson Novoa, che a vario titolo hanno prestato la loro collaborazione, e al prof. Marco Tangheroni per i suoi consigli e suggerimenti.

-Vicente Salavert Roca, Cerdeña y la expansión mediterránea de la Corona de Aragón. 1297-13I4, II, Madrid, 1956, doc. 36, pp. 45-46; si tratta della più antica attestazione fino ad oggi nota dell'esistenza di un consolato catalano in Sardegna. Riguardo all'elezione dei consoli d'Oltremare, il sovrano catalano-aragonese avrebbe dovuto limitarsi, secondo il privilegio di Giacomo I, a ratificare le scelte dei consiglieri barcellonesi.

"Anuario de Istudios Medievales". 29 (1999)
} 
L'esito della vicenda non ci è noto, tuttavia gli studiosi che se ne sono occupati mi sembra siano propensi, pur con le dovute cautele, a ritenere priva di riscontri effettivi la nomina di questo sconosciuto pisano e a considerare un episodio isolato l'intromissione di Giacomo II nelle questioni inerenti i consolati catalani in Sardegna ${ }^{3}$. Qui, come del resto in Sicilia, durante il XIV secolo nel conferimento degli incarichi consolari sarebbe prevalsa la volontà dei consiglieri barcellonesi, che per tali uffici si sarebbero serviti per lo più di personaggi di loro fiducia ${ }^{4}$.

Riguardo all'eventuale arbitrarietà dell'intervento di Giacomo, si deve però tener presente che, come validamente ribadito in anni recenti da Regina Sáinz de la Maza, il famoso privilegio del Conquistatore non sempre veniva rispettato alla lettera: a Pisa, per esempio, in diverse occasioni il titolo di console fu attribuito da Pietro III o, successivamente, da Giacomo $\mathrm{II}^{5}$, e lo stesso dicasi per i consolati di Tunisi e Bugia durante il periodo di regno di Giacomo I ${ }^{6}$.

In questo panorama l'iniziativa del sovrano catalano-aragonese del 1301 riguardo a Castel de Castro potrebbe dunque apparire non del tutto anomala, e forse soltanto nel 1321, anno a cui risalgono alcuni speciali decreti emanati da Giacomo II a favore di Barcellona in vista della conquista del regno di Sardegna e Corsica, i consiglieri della città comitale si riappropriarono definitivamente anche in Sardegna della prerogativa di nomina dei consoli?.

${ }^{3}$ Luisa D'ARIEnzo, Una nota sui consolati catalani in Sardegna nel secolo XIV, in "La Península Ibérica y el Mediterráneo centro-occidental (siglos XII-XV)", I Congreso Internacional de Historia Mediterránea (Palma de Mallorca, 1973), Barcelona-Roma, 1980, pp. 594-595 e 609; Regina SáINZ DE LA MAZA LASOlI, Il consolato dei Catalani a Pisa durante il regno di Giacomo II d'Aragona. Notizie e documenti, "Medioevo. Saggi e Rassegne", 20 (1995), pp. 200-201.

${ }^{4}$ Sui consolati catalani in Sicilia si veda da ultimo Josefina MuTGÉ VIVES, Il Consell di Barcellona nell'ambito della politica siciliana di Giacomo II d'Aragona, "Medioevo. Saggi e Rassegne", 20 (1995), pp. 223-250.

${ }^{5}$ Regina SÁINZ DE LA MAZA LASOLI, Il consolato dei Catalani, cit. Nell'articolo in questione l'autrice non sottovaluta comunque la querelle fra Giacomo e i consiglieri di Barcellona sul consolato cagliaritano.

${ }^{6}$ Charles-Emmanuel DufourcQ, Les consulats catalans de Tunis et de Bougie au temps de Jacques le Conquérant, "Anuario de Estudios Medievales”, 3 (1966), pp. 469-479.

${ }^{7}$ Il 10 dicembre 1321 Giacomo II concesse alla città di Barcellona "la prerogativa di eleggere consoli in Sardegna e in Corsica e di allontanarli dall'incarico a loro arbitrio" nonché "l'esenzione da qualsiasi tipo di imposta su tutte le mercanzie in importazione o in esportazione dalla Sardegna, dalla Corsica e dalle isole adiacenti, in cambio del prestito concessogli dal Consiglio della città di millecinquecento lire barcellonesi, necessarie per la conquista dell'isola" 
Per quanto concerne invece l'eventualità che un pisano nei primi anni del Trecento potesse essere chiamato a difendere gli interessi dei mercanti catalani nell'isola, ipotesi in passato ritenuta poco probabile, sarà sufficiente rammentare che la giurisdizione del console dei Catalani a Pisa in quello stesso periodo includeva anche Castel di Castro, almeno nel caso di Bartolomeo Sismondi, Bundo del Campo, Puccio e Jacopo Lanfreducci ${ }^{8}$. E sebbene allo stato attuale degli studi non risulti chiaro a questo proposito il genere di vincolo che univa Pisa a Castel di Castro, in quanto sia nella lettera dei consiglieri di Barcellona precedentemente citata sia nel documento che esaminerò poi sembra sussistere in Sardegna un autonomo consolato dei Catalani $^{9}$, è comunque evidente che accedere al suddetto ufficio consolare non costituiva affatto per un pisano una possibilità remota.

Intanto, come già accennato, anche un documento attualmente custodito in una delle serie cancelleresche dell'Archivio della Corona d'Aragona perviene ad accreditare la validità di tale ipotesi.

Si tratta di un testo redatto a Castel di Castro nell'aprile 1320 e da qui trasmesso a Barcellona per denunciare un atto di pirateria compiuto da alcuni Genovesi residenti a Savona a danno di due mercanti catalani, episodio avvenuto nel marzo dello stesso anno in prossimità di Capoterra, nel

(Luisa D’ARIEnzo, Una nota sui consolati, cit., pp. 595-596).

${ }^{8}$ Il Sismondi, Bundo del Campo e Puccio Lanfreducci furono eletti consoli rispettivamente negli anni 1292, 1302 e 1304; non sappiamo a quando risale il decreto di nomina di Jacopo, che risulta però console nel periodo 1317-1320 (Regina SáINZ DE LA MAZA LASOLI, Il consolato dei Catalani, cit., pp. 202 e 205-207).

${ }^{9} L$ 'istituzione di tale consolato potrebbe essere dovuta alla non breve distanza da Pisa, all'elevato numero di Catalani presenti in Castello nonché alle particolari condizioni socioeconomiche dell'universitas cagliaritana, il cui nucleo di "borghesi" già nel secolo precedente aveva dato segni di insofferenza verso la madrepadria. Su quest'ultimo tema si veda in particolare Sandro PETRUCCI, Forestieri a Castello di Castro in periodo pisano, in "Commercio, finanza, funzione pubblica. Stranieri in Sicilia e in Sardegna nei secoli XIII-XV", a cura di Marco Tangheroni, Napoli, 1989, pp. 219-259. Sul significato del termine burgensis, oltre al suddetto studio di Petrucci, si vedano anche Francesco ARTIZZU, Civis e burgensis nella terminologia giuridica sardo pisana (appunti per un discorso da sviluppare ulteriormente), "Annali della Facoltà di Magistero dell'Università di Cagliari”, n.s., V, II (1981), pp. 3-8; Marco TANGHERONI, La città dell'argento. Iglesias dalle origini alla fine del Medioevo, Napoli, 1985 , pp. $222-228$. 
meridione della Sardegna ${ }^{10}$. L'interesse del documento, oltre che nella descrizione minuziosa dell'accaduto e nella menzione di merci e percorsi dei malcapitati coinvolti, sta soprattutto nell'escatocollo, dove si susseguono le firme dei mercanti catalani e maiorchini che confermano da Castel di Castro la veridicità del racconto. In cima a tutti, estensore della missiva su richiesta del tortosano Domingo Fuster, compare il loro console: Neri Moxeriffo, indiscutibilmente un pisano.

Egli non afferma ciò in modo esplicito, tuttavia il nome rivela la sua provenienza e rende possibile l'identificazione con un personaggio segnalato a Castel di Castro nei primi decenni del XIV secolo da diverse fonti documentarie.

Del Moxeriffo, appartenente ad una nota famiglia popolare la cui presenza è testimoniata a Pisa fin dagli anni $1240-1260^{11}$, padre di Giovanni e Piero e forse sposato con una tale monna Nuta ${ }^{12}$, sappiamo che possedeva alcune case in Castello ${ }^{13}$, che era in affari con Gaddo Gambacorta ${ }^{14}$, Cecco della Cantera, Bonaccorso Gambacorta e Guido Papa ${ }^{15}$, e che nel marzo 1317 figurava tra i compositores introitum et reddituum iudicatus

\footnotetext{
${ }^{10}$ Archivo de la Corona de Aragón (ACA), Cancillería (C), Cartas Reales de Jaime II (CR de Jaime II), caja 52, c. 6399.

"Marco Tangheroni, Gli Alliata. Una famiglia pisana del Medioevo, Padova, 1969, p. 6.

'I familiari del Moxeriffo sono citati in una lettera scritta dal mercante Mone Acciaio che esaminerò poi (ACA, C, CR de Jaime II, caja 61, c. 7547). Giovanni e Piero compaiono anche in un documento relativo ai risarcimenti effettuati dagli ufficiali del regno di Sardegna a favore di quei Pisani le cui case in Castello, dopo il secondo trattato di pace stipulato fra Pisa e la Corona d'Aragona il 25 aprile 1326, erano state assegnate a sudditi iberici. In quest epoca Neri Moxeriffo è già deceduto) (Rafael CONDE Delgado DE MOLINA; Antonio María ARaGó CABAÑAs, Castell de Câller. Cagliari catalano-aragonese, Cagliari, 1984, pp. 159-160). Nel febbraio del 1324 risulta camerlengo di Villa di Iglesias un tale Giovanni Moxeriffo, verosimilmente identificabile con il suddetto figlio di Neri (Carlo BAUDI DI VeSME. Coder Diplomaticus Ecclesiensis, Torino, 1877, doc. XXIX, coll. 382-383, doc. XXXVI, col. 394).

${ }^{13}$ Rafael Conde Delgado de Molina; Antonio María Aragó Cabañas, Castell de Càller, cit., pp. 55, 62, 71, 80, 159-160; Carlo LiVI, La popolazione della Sardegna nel periodo aragonese, "Archivio Storico Sardo", XXXIV, II (1974), p. 81; Maria Bonaria URBAN, Nuovi elementi di storia urbana nel regno di Sardegna: dalla fondazione di Bonaria al popolamento catalano di Castel di Cagliari "Anuario de Estudios Medievales", 27, II (1997), pp. 846 e 863 EAD., Da Bonaria a Castel di Cagliari: programma politico e scelte urbanistiche nel primo periodo del regno di Sardegna catalano-aragonese, "Medioevo. Saggi e Rassegne", 22 (1997), pp. 129 e 145-146.

${ }^{14}$ Emilio CRISTIANI, Nobiltà e popolo del comune di Pisa. Dalle origini del podestariato alla signoria dei Donoratico, Napoli, 1962, p. 465; il documento in questione è del 1301.

${ }^{15}$ Carlo Baudi DI Vesme, Codex Diplomaticus, cit., doc. V, coll. 340-341; il documento risale al 1314
} 
kallaretani pro comuni pisano ${ }^{16}$. Sappiamo inoltre che pochi mesi dopo tale data, in maggio, i curatori dell'eredita di Neri da Riglione, un facoltoso mercante pisano deceduto in Sardegna, gli consegnavano più di 1400 starelli di grano ${ }^{17}$.

Prima dello sbarco dell'infante Alfonso d'Aragona a Palma di Sulci, nel giugno 1323, come molti altri suoi concittadini, era forse definitivamente rientrato in Toscana, pur mantenendo saldi legami con l'isola. Lo confermerebbero sia l'anzianato conferitogli nel luglio dello stesso anno ${ }^{18}$, sia una lettera a lui spedita da Castel di Castro in ottobre dal mercante Mone Acciaio $^{19}$, il cui tenore mi pare non lasci dubbi sull'avvenuto trasferimento del Moxeriffo a Pisa e sulla continuità dei suoi negozi in Sardegna. Mone Acciaio infatti, oltre ad avvertire l'amico dell'invio di un carico d'orzo ${ }^{20}$, nel

${ }^{16}$ Francesco ArTizzu, L'Aragona e i territori pisani di Trexenta e Gippi, "Annali delle Facoltà di Lettere, Filosofia e Magistero dell'Università di Cagliari”, XXX (1966-1967), pp. 404-405.

${ }^{17}$ Francesco ARTIZZU, Neri da Riglione borghese di Cagliari, in Francesco ARTIZZU, Pisani e Catalani nella Sardegna medioevale, Padova, 1973, p. 49. Nell'affaire dell'eredità da Riglione il Moxeriffo compare anche, per conto dei familiari del defunto, come depositario della somma di 600 lire di denari aquilini minuti che Cecco Alliata e Vanni da Riglione sono incaricati di restituire a Lippo Alliata (Documenti inediti relativi ai rapporti economici tra la Sardegna e Pisa nel Medioevo, a cura di Francesco Artizzu, II, Padova, 1962, doc. 14, pp. 39-43).

${ }^{18}$ Emilio Cristiani, Nobiltà e popolo, cit., p. 465. Sugli anzianati attribuiti alla famiglia Moxeriffo vedi anche Bruno CASINI, Il "Priorista" $e$ i "Libri d'oro" del Comune di Pisa, Firenze, 1986, p. 100

${ }^{19} \mathrm{ACA}, \mathrm{C}, \mathrm{CR}$ de Jaime II, caja 61 , c. 7547 . Il mittente credo possa essere identificato con il Mone Acciaio che nel febbraio 1317 ricevette da Cecco Alliata e Vanni da Riglione 186 lire di denari aquilini minuti e 837 starelli di grano, a lui dovuti da Neri da Riglione (Documenti inediti, cit., II, docc. 6 e 14, rispettivamente pp. 11-15 e 39-43). Ritroviamo lo stesso personaggio fra gli anziani di Castel di Castro che il 16 giugno 1324 eleggono il notaio Percivalle loro rappresentante nelle trattative di pace con l'infante Alfonso d'Aragona (Francesco ARTIZZU, In margine al trattato di pace pisano-aragonese del 1324. Le procure al plenipotenziario Bene da Calci ed al notaio Percivalle, in Francesco ARTIZZu, Pisani e Catalani, cit., doc. II, pp. 131-132), e in un elenco di sospetti fornito nell'agosto 1326 dall'infante Alfonso a Bernat de Boxadors e ad altri ufficiali regi (Rafael CONDE DELGADO DE Molina: Antonio María ARagó Cabañas, Castell de Càller, cit., p. 207: vedi anche Marco TANGHERONI, Alcuni aspetti della politica mediterranea di Giacomo II d'Aragona alla fine del suo regno, "Annali delle Facoltà di Lettere, Filosofia e Magistero dell'Università di Cagliari", XXXII (1969), doc. III, pp. 154-155). Sulla lettera di Mone Acciaio a Neri Moxeriffo, probabilmente intercettata dai Catalani, si vedano anche Antonio ARriBAS PALAU, La conquista de Cerdeña por Jaime II de Aragón, Barcelona, 1952, p. 213; Carlo LIVI, La popolazione della Sardegna, cit., p. 79.

${ }^{20} \mathrm{Si}$ tratta di 93 staia d'orzo inviate al Moxeriffo, tramite Lore Sardo, da parte di Guido Caccialoste, probabilmente lo stesso personaggio che, a fronte delle reiterate proibizioni di Alfonso IV di ammettere a Castel di Castro e nelle appendici quanti non appartenessero alla nazione catalana, nel 1329 ricevette l'autorizzazione regia di risiedere e commerciare nel suddetto Castello (Rafael Conde Delgado DE Molina; Antonio María Aragó Cabañas, 
commentare novità e avvenimenti dell'isola sembra a lui rivolgersi come a persona lontana, chiamata ad intervenire in altro luogo sull'andamento degli affari sardi ${ }^{21}$.

Fra le possibili motivazioni che avrebbero indotto Giacomo II ad assegnare a cittadini pisani l'ufficio di console dei Catalani a Pisa e a Castel di Castro, gli storici hanno supposto da un lato uno scarso interesse da parte dei mercanti iberici di insediarsi a Pisa per svolgere un incarico giudicato poco remunerativo ${ }^{22}$, dall'altro, relativamente alla Sardegna, l'intenzione del sovrano di ricompensare personaggi a lui devoti ${ }^{23}$ o altrimenti di far fronte, anche in quest'ambito, alla difficile situazione politico-diplomatica creatasi negli ultimi anni del XIII secolo ${ }^{24}$. L'infeudazione del regno di Sardegna e Corsica a Giacomo II da parte di Bonifacio VIII nel 1297 aveva infatti in certo modo compromesso le "secolari amichevoli relazioni" 25 con il comune dell'Arno e instaurato fra l'Aragona e Pisa uno stato di latente, malcelata ostilità.

Tuttavia, nell'esame di tale problematica non va forse trascurata la sottile abilità da parte catalana di adattarsi alle circostanze del luogo in cui si trovavano ad operare i mercanti della Corona e quindi l'accortezza di preferire un "locale" ogniqualvolta le suddette circostanze indicassero tale

Castell de Càller, cit., p. 24).

${ }^{21}$ In un documento edito da Rafael Conde relativo all'espulsione dei Pisani da Castello, atto di poco posteriore alla pace del 1326 , Neri Moxeriffo figura per tre volte genericamente stabilito a Pisa e soltanto in un'annotazione si specifica che "és foragitat per sospitós", cioè espulso in quanto sospetto (Rafael Conde Delgado de Molina; Antonio María Aragó Cabañas, Castell de Câller, cit., p. 62). In Sardegna rimase forse un parente di Neri, Lemmo Moscerifi, appaltatore del diritto di "mensuratura della vena" negli anni 1331 e 1332 (Marco TANGHERONI, La città dell'argento, cit., p. 315).

${ }^{22}$ Regina SÁINZ DE LA MAZA LASOLI, Il consolato dei Catalani, cit., p. 200.

${ }^{23}$ Raffaele DI TUCCI, I consoli in Sardegna (sec. XII-XVII), "Archivio Storico Sardo”, VIII (1912), p. 86.

${ }^{24}$ Sandro Petrucci, Forestieri a Castello di Castro, cit., p. 247.

${ }^{25}$ Marco Tangheroni, Pisa e la Corona d'Aragona, in "Momenti di storia medioevale pisana. Discorsi per il giorno di S. Sisto", a cura di Ottavio Banti e Cinzio Violante, Pisa, 1991, p. 141. 
scelta come la più conveniente ${ }^{26}$. In particolare a Castel di Castro, dove $\mathrm{i}$ rapporti di collaborazione commerciale fra Pisani e Catalani non erano infrequenti ${ }^{27}$, neppure a livello istituzionale ${ }^{28}$, ma dove la situazione andava complicandosi per questi ultimi sia per il risentimento cagionato a Pisa dalla citata investitura papale sia per una più marcata tendenza all'accentramento da parte dell'oligarchia comunale ${ }^{29}$, un pisano avrebbe forse con miglior esito provveduto a salvaguardare le fortune dei Catalani in Sardegna.

Se così fosse, non desterebbe meraviglia l'elezione di Neri Moxeriffo, rientrando anzi nella logica di una concreta valutazione. Ricco mercante, cittadino influente a Pisa e in Castello, esperto dell'ambiente mercantile iberico, come attesterebbe la sua vicinanza ai più intraprendenti

\footnotetext{
${ }^{26} \mathrm{Su}$ tale questione si rimanda specificamente ad un intervento di Marco Tangheroni al "III Convegno Internazionale di Studi Colombiani" tenutosi a Genova nel 1977 (Genova, 1979, pp. 61-62).

${ }^{27}$ Marco Tangheroni ha in più occasioni segnalato esempi di società fra operatori pisani $\mathrm{e}$ catalani costituitesi negli anni precedenti la spedizione dell'infante Alfonso in Sardegna, insistendo poi sul fatto che non si possa parlare neanche per il periodo successivo al 1326 di un definitivo allontanamento di Pisa dalle risorse economiche sarde. Nonostante le relazioni non proprio idilliache con $\mathrm{i}$ re d'Aragona e un oscillante riavvicinamento a Genova, i Pisani riuscirono infatti a mantenere saldi i loro interessi commerciali nell'isola e nei mercati iberici. Su tali problematiche, oltre alle opere già citate di Tangheroni, si vedano dello stesso autore: Pisa e la Corona d'Aragona, in "La Corona de Aragón en el siglo XIV", VIII Congreso de Historia de la Corona de Aragón (Valencia, 1967), II, Valencia, 1973, pp. 145-175; Note sui rapporti tra Pisa, l'Aragona e Genova all'epoca di Alfonso il Benigno (1327-1336), in I Congresso Storico Liguria-Catalogna (Ventimiglia-Bordighera-Albenga-Finale-Genova, 1969), Bordighera, 1974, pp. 177-182; Sui rapporti tra il comune di Pisa e il regno d'Aragona nella seconda metà del XIV secolo, "Studi Sardi”, XXI (1968-1970), pp. 80-94; Politica, commercio, agricoltura a Pisa nel Trecento, Pisa, 1973, pp. 107-126 e 131-143; Due documenti sulla Sardegna non aragonese del Trecento, "Medioevo. Saggi e Rassegne", 2 (1976), pp. 27-64. Sulla presenza dei mercanti catalani in Sardegna, attestata da antica data (prima metà del XIII secolo). si rimanda a Carmen BATLLE, Noticias sobre los negocios de mercaderes de Barcelona en Cerdeña hacia 1300, in "La Sardegna nel mondo mediterraneo". I Convegno Internazionale di Studi Geografico-Storici (Sassari, 1978), II, a cura di Manlio Brigaglia, Sassari, 1981, pp. 277-289; Joan F. CABESTANY FORT, I mercanti catalani e la Sardegna, in "I Catalani in Sardegna”, a cura di Jordi Carbonell e Francesco Manconi, Cinisello Balsamo, 1984, pp. 25-30.

${ }^{28}$ Sandro Petrucci segnala per gli anni successivi alla battaglia della Meloria (1284) l'utilizzo di navi catalane e maiorchine da parte del comune di Pisa (Sandro PETRUCCl, Forestieri a Castello di Castro, cit., pp. 245-246; ID., Tra Pisa e Maiorca: avvenimenti politici e rapporti commerciali nella prima metà del XIV secolo, in XIII Congrés d'Història de la Corona d'Aragó (Palma de Mallorca, 1987), I, Palma de Mallorca, 1989, p. 139).

${ }^{29} \mathrm{Nel} 1313$ le autorità comunali pisane estesero anche ai Catalani il divieto di divenire "borghesi" di Castel di Castro. Secondo Sandro Petrucci si sarebbe trattato di una misura atta, più che a limitare le iniziative commerciali di costoro, ad impedire un'eventuale partecipazione alla vita pubblica da parte di quanti avessero acquisito tale status (Sandro PETRUCCI, Forestier a Castello di Castro, cit., pp. 231-234 e 247; ID., Tra Pisa e Maiorca, cit., p. 143).
} 
mercanti e banchieri pisani attivi in Sardegna, gli Alliata e i Gambacorta, di cui sono note le relazioni societarie con Catalani e Maiorchini ${ }^{30}$, Neri sembra disporre di tutti i requisiti necessari per svolgere con successo il suo ufficio di console.

Il resoconto di quanto accaduto ai mercanti Domingo Fuster e Bertran Thevissa redatto da Neri Moxeriffo apre un chiaro spiraglio sul mondo cosmopolita degli affari che ruotavano attorno alla rocca di Castel di Castro, felicemente collocata al centro delle rotte mediterranee e degli interessi commerciali di Pisani e Catalani. Allo stesso modo si rivela significativa per comprendere quanto stava avvenendo in Sardegna nei mesi successivi allo sbarco delle truppe catalano-aragonesi a Palma di Sulci la lettera scritta da Mone Acciaio nell'ottobre del 1323. Sono trascorsi soltanto tre anni, eppure i cambiamenti in atto risultano già evidenti: Mone fa il conto delle provviste a Castello e a Villa di Chiesa, valuta con apprensione le novità giunte dalla Sicilia, riflette con un misto di malinconia e scetticismo su quel legame fra Castello e la madrepatria tanto declamato dagli ambasciatori pisani che nel 1309 si recarono alla corte aragonese ${ }^{31}$, per poi chiedere all'amico di mostrare la sua lettera anche a messer Bonagiunta da Vico, "che so che porta amore a questa terra, acciò che s'aoperi giusto suo podere che noi non periamo" 32 .

Nell'autunno del 1323 Mone Acciaio vive ormai in una città in procinto di essere assediata.

E se le conseguenze del gesto di Bonifacio VIII non pregiudicarono per sempre le relazioni fra il comune di Pisa e la Corona d'Aragona, né tanto meno arrestarono l'operosità dei più avveduti mercanti toscani, abili nel modificare i percorsi di merci e capitali, per la comunità di Castel di Castro, parva Pisa ${ }^{33}$, l'arrivo dell'infante Alfonso in Sardegna segnò per davvero il termine di una lunga storia.

\footnotetext{
${ }^{30}$ Sandro PETRUCCI, Forestieri a Castello di Castro, cit., pp. 246-247. Riguardo agli Alliata, si ricordi che nel 1320, anno del consolato di Neri Moxeriffo, Betto era castellano di Castel di Castro insieme a Iacopo Fagioli (Marco TANGHERONI, Gli Alliata, cit., p. 45).

${ }^{31}$ Sandro PETRuCCI, Forestieri a Castello di Castro, cit., p. 223.

${ }^{32} \mathrm{ACA}, \mathrm{C}, \mathrm{CR}$ de Jaime II, caja 61, c. 7547.

${ }^{33}$ Sandro PETRuCCI, Forestieri a Castello di Castro, cit., p. 223.
} 


\section{APPENDICE DOCUMENTARIA}

1320, aprile, 12. Castel di Castro.

Neri Moxeriffo, console dei Catalani a Castel di Castro, insieme ad alcuni mercanti catalani e maiorchini denuncia un atto di pirateria compiuto il 5 marzo 1320 da alcuni Genovesi a danno di Domingo Fuster e Bertran Thevissa nei pressi di Capoterra.

Archivo de la Corona de Aragón, Cancillería. Cartas Reales

de Jaime II, caja 52, c. 6399.

Serta cossa sia et maniffesta a totz cels qui esta carta veuran que h[a] requesta d'en Domingo Fuster, ciutadà de Tortossa, fem aquesta pressent charta, con sia serta cossa que en Domingo Fuster et en Bertran Thevissa vinguesen de les partz de Sisilia, del port de Tràpena, en I leyn de bandes de Arnau de Soler, ciutadà de Barcelona, enant en son viatge ha Malorcha, et foren en la illa de Serdey(n)a, a Caboterra. Trobaren aquí I leyn de Jenovezes de Sahona al V jorns del mes (de) mars a l'entrada, e conbateren lo dit leyn de Arnau de Soler et preseren-lo per forssa de batalla et ausiyeren-hi lo demun dit Bertran Thevissa et preseren lo leyn ab lo dit Domingo Fuster et ab totz cels qui dintra heren. E robaren lo dit Domingo Fuster de tot quant el havia, que no li lexaren vida ha I jorn, e menaren-lo s'en pres el et elz altres ab lo dit leyn fins ha Terranova, qui és en la illa ha $\mathrm{CC}$ milles de Chàler, et aquí possaren-los en terra. E levaren al demunt dit Bertran Thevissa XXI esclau(s) sareyns ab d'altres robes moltes, axí con és chasseria et robes de Barberia et armes et arnès, qui heren romazes del seu leyn, lo qual havia venut ha Marssara en Sisilia.

E a testimoni de viritat sobre les demun dites chozes, nós conssoll Neri Moxeriffo en Castell de Chàler per los Catellans, ab los mercaders catalans escrivim de nostres mans et possam-hi nostres segells.

Escrita la carta en Castell de Chàler en l'ayn de Nostre Senyor $\mathrm{M}^{\circ} \mathrm{CCC}^{\circ} \mathrm{XX}$, ha XII jorns del mes de abrill.

Yo en Guillem de [Onjatis] ${ }^{\prime}$, merqader de Malorcha, escriu axy de ma mà et en testimony de verytat posi ych mon segel.

\footnotetext{
'Parola di difficile lettura.
} 
Ego Arnau de Soler, petró del demun dit leyn, dic et fas testimoni que.l dit Bertran Tivisa avia en lo dit leyn totes aqestes coses et encara més, et per so com jo no avia segel, pregé el seynhor en Guerao Bertran de Barselhona que'm prestàs lo seu.

Yo en Maymon Ponts de Sen Feliu de Guíxols fas testimoni de les cosas damon escritas et posi mon sagel.

Jo Antich Ses Hiles, mercader de Malorcha, testimoni sobre les demun dites cozes et pozi mon segell.

Jo en Jacme Lunell, patró de leyn de Barcellona, testimoni sobre les demun dites cozes et pozi mon segell.

Jo Arnau Sa Guardiola fas testimoni que.s serts que.l demunt dit leny fo pres e.ls homens robats et denafrats de I leny de Janovezes et pozi mon segel.

Jo en Berenguer Ses Planes, merchader de Barsalona, fas testimoni sobre les demon dites coses et posy mon sagel.

\section{RÉSUMÉ}

Un document se trouvant actuellement aux Archives de la Couronne d'Aragon -à Barcelone- confirme qu'au début du XIV ${ }^{\mathfrak{c}}$ siècle, il y avait la possibilité pour un pisan de devenir consul des catalans à Castel di Castro, et ce malgré l'hostilité qui existait alors entre la ville de Pise et la Couronne d'Aragon. Celle-ci était dûe à la concession féodal du Règne de Sardaigne et de Corse instaurée en 1297 par le Pape Boniface VIII en faveur de Jacques II d'Aragon. L'article en question examine les raisons qui pourraient amener les catalans à attribuer cette charge à un pisan et comporte plusieurs notes biographiques sur le consul Neri Moxeriffo.

\section{SUMMARY}

A document to be found in the Archive of the Crown of Aragon in Barcelona confirms that in spite of the hostility between the city of Pisa and Aragon by means of the Kingdom of Sardinia and Corsica conceded by Pope Boniface VIII to James II of Aragon (1297), in the first decades of the XIV century it was possible for a pisan to become consul of the Catalans at Castel di Castro. In the article in question the possible motivations which induced the Catalans to entrust a Pisan with the aforementioned task are mentioned along with an up-to date bibliography about the consul Neri Moxeriffo. 